\title{
HOMOMORPHISM THEORIES FOR UNIVERSAL ALGEBRAS
}

\author{
by HANS-JÜRGEN HOEHNKE \\ (Received 23rd September 1967)
}

\section{Introduction}

It is well-known that a homomorphism $\phi(A \rightarrow B)$ between groups $A$ and $B$ induces a homomorphism $\phi^{*}(Z A \rightarrow Z B)$ between the corresponding group rings $Z A$ and $Z B$ over the ring of integers $Z$. The identical congruence $O$ on $B$ and the unit element $e_{B}$ of $B$ can be characterised by the equations $x-y=0$ and $x-e_{B}=0(x, y \in B)$ respectively. Similarly the congruence $\Gamma_{\phi}$ corresponding to $\phi$ and the corresponding normal subgroup of $A$ are

$$
\left\{(x, y) \in A^{2}=A \times A,(x-y) \phi=0\right\}
$$

and $\left\{x \in A^{1}=A,\left(x-e_{A}\right) \phi=0\right\}$ respectively. Suppose that instead of groups we choose any class $L$ of algebraic systems each with a binary operation (groupoids), and replace $x-y$ or $x-e_{A}$ by a fixed polynomial $P(x)$, where $x=\left(x_{1}, \ldots, x_{n}\right)$, defined for all groupoid rings $Z A(A \in L)$. Then the $P$-kernel of the homomorphism $\phi$ from $A$ to $B(A, B \in L)$ can in general be defined by $N_{\phi}=\left\{(x) \in A^{n}, P(x) \phi=0\right\}$. It seems desirable that $(a)$ the $P$-kernels can be characterised, independently of homomorphisms, as those subsets of $A^{n}$ which are $P$-closed with respect to an appropriate closure operation $C l_{P},(b)$ given any $P$-closed set $N \subseteq A^{n}$, the $P$-factor groupoid $(A / N)_{P}$ and the homomorphism $\eta: A \rightarrow(A / N)_{P}$ with $P$-kernel $N$ correspond canonically to $N$ and $(c)$ the correspondence $\Gamma_{\phi} \rightarrow N_{\phi}$ is monotone, as in the case, for example, of semigroups $A$ with zero element $O_{A}$, with respect to the polynomial $x-O_{A}$.

Following this approach, G. Losey (6) has found that, by specifying $P$, various known results on kernels for groupoids can be included in the theory. However, at a crucial point ((6), p. 132, proof of Theorem 3.4), Losey seems to have confused the zero element of the groupoid ring $Z(A / N)_{P}$ with the zero element of the factor ring $Z A$ with respect to the kernel $\{0\} \eta^{*-1}$ of the mapping $\eta^{*}: Z A \rightarrow Z(A / N)_{P}$. Thus the proofs of all the theorems in (6) concerning the $P$-factor groupoid $(A / N)_{P}$ and the identification of $P$-kernels with $P$-closed sets contain a gap. The example of the class of all groupoids with $P(x)=x$ as the polynomial shows easily that the theorem in question is certainly not valid in the generality asserted. In this note it is shown that the gap can be closed if the polynomial $P$ satisfies a crucial but necessary and sufficient condition. In many important cases this condition is fulfilled. 
At the same time the concept of groupoid ring is so generalised that any (not necessarily binary) algebra can be included. Then $P$ can be defined by a positive formula in a first order predicate calculus with identity.

The author gratefully acknowledges the assistance of Mr G. Martin and Professor E. M. Patterson of Aberdeen in translating and submitting the paper.

\section{The algebra-ringoid $Z A$}

Let $L$ be a class of universal algebras of the same finitary type $\Omega$ with ground operations $\alpha \in \Omega$. If $A \in L$, let $Z A$ be the aggregate of formal sums $\sum_{a \in A} \mu(a) a$, with coefficients $\mu(a)$ from the ring of integers $Z$, such that $\mu(a)=0$ for all but a finite number of elements $a \in A$. Two such sums with coefficients $\mu(a), v(a)$ are equal precisely when $\mu(a)=v(a)$ for each $a \in A$. Given an $n$-ary operation $\alpha \in \Omega$, where $n \geqq 1$, we write

$$
\alpha\left(\sum_{a \in A} \mu_{1}(a) a, \ldots, \sum_{a \in A} \mu_{n}(a) a\right)=\sum_{a_{1} \in A} \ldots \sum_{a_{n} \in A} \mu_{1}\left(a_{1}\right) \ldots \mu_{n}\left(a_{n}\right) \alpha\left(a_{1}, \ldots, a_{n}\right)
$$

and for each constant operation $\alpha=a \in A$, we write $\alpha=1 a$. Then $Z A$ becomes an algebra of the same type as $A$ : that is, of type $\Omega$. If we write

$$
\sum_{a \in A} \mu(a) a+\sum_{a \in A} v(a) a=\sum_{a \in A}(\mu(a)+v(a)) a
$$

then $Z A$ becomes an additive abelian group. Henceforth we shall regard $Z A$ as an algebra of the type $\Omega \cup\{+\}$ and call this algebra the algebra-ringoid of $A$ over $Z$. This clearly generalises the concept of groupoid ring. If $s, s_{1}, \ldots, s_{n} \in Z A$, $n \geqq 1$ and $\alpha \in \Omega$, the distributive law holds in the generalised form

$$
\begin{aligned}
\alpha\left(s_{1}, \ldots, s_{i-1}, s_{i}+\right. & \left.s, s_{i+1}, \ldots, s_{n}\right) \\
& =\alpha\left(s_{1}, \ldots, s_{i-1}, s_{i}, s_{i+1}, \ldots, s_{n}\right)+\alpha\left(s_{1}, \ldots, s_{i-1}, s, s_{i+1}, \ldots, s_{n}\right) .
\end{aligned}
$$

In general, we shall call an algebra $R$ of the type $\Omega \cup\{+\}$ a ringoid whenever the (+)-structure $R^{+}$of $R$ determines an abelian group and the above distributive law is satisfied. The $\Omega$-structure of $R$ will be denoted by $R^{\Omega}$. This concept of ringoid is not the same as that of (2), nor is it the same as the concept of $\Omega$ ringoid of (3). Since our ringoids are closely related to rings, they could also be called $\Omega$-rings. From the distributive law with $s=0$ it follows that

$$
\alpha\left(s_{1}, \ldots, s_{i-1}, 0, s_{i+1}, \ldots, s_{n}\right)=0 \quad(n \geqq 1) .
$$

The correspondence $b \rightarrow \sum_{a \in A} \mu(a) a$, where $\mu(a)=1$ if $a=b$ and $\mu(a)=0$ if $a \neq b$, determines an isomorphism of $A$ into $(Z A)^{\Omega}$; we may therefore identify $b \in A$ with its image in $Z A$.

Any homomorphism $\phi(A \rightarrow B)$ from $A$ into $B$, where $A, B \in L$, can be extended linearly to a ringoid-homomorphism $\phi^{*}(Z A \rightarrow Z B)$ by writing

$$
\left(\sum_{a \in A} \mu(a) a\right) \phi^{*}=\sum_{a \in A} \mu(a)(a \phi)=\sum_{b \in B}\left(\sum_{\substack{a \in A \\ a \phi=b}} \mu(a) b .\right.
$$


Conversely, any ringoid-homomorphism $\phi(Z A \rightarrow R)$ of $Z A$ into a ringoid $R$ induces a homomorphism of the algebra $A \subseteq(Z A)^{\Omega}$ into a subalgebra of $R^{\Omega}$. If $\phi\left(R_{1} \rightarrow R_{2}\right)$ is a ringoid-homomorphism, then

$$
\{0\} \phi^{-1}=\left\{s \in R_{1}, s \phi=0\right\}
$$

is called the kernel of $\phi$. The kernel $\{0\} \phi^{*-1}$ of the linear extension $\phi^{*}(Z A \rightarrow Z B)$ of $\phi(A \rightarrow B)$ is also called the $r$-kernel $K_{\phi}$ of the homomorphism $\phi$.

A non-empty subset $K$ of a ringoid $R$ is called a subringoid of $R$ if $K$ is a ringoid with respect to the operations defined in $R ; K$ is called an ideal of $R$ if for all $s \in K, s_{1}, \ldots, s_{n} \in R$ and all $\alpha \in \Omega$, we have

$$
\alpha\left(s_{1}, \ldots, s_{i-1}, s, s_{i+1}, \ldots, s_{n}\right) \in K \quad(n \geqq 1)
$$

and $K$ is a subgroup of $R^{+}$. The kernel $\{0\} \phi^{-1}$ of a ringoid-homomorphism $\phi\left(R_{1} \rightarrow R_{2}\right)$ is an ideal of $R_{1}$. Conversely, any ideal $K$ of a ringoid $R$ corresponds canonically to a factor ringoid $R / K=\{s+K, s \in R\}$ and a homomorphism $R \rightarrow R / K$ defined by $s \rightarrow s+K$. For $\phi\left(R_{1} \rightarrow R_{2}\right), R_{1} \phi$ is a subringoid of $R_{2}$ and $R_{1} \phi \cong R_{1} / K$, where $K=\{0\} \phi^{-1}$. If $A \in L$, if $K$ is an ideal of $Z A$ and $\eta(Z A \rightarrow(Z A) / K)$ is the canonical homomorphism, then $\eta$ induces a homomorphism $\bar{\eta}$ of $A \subseteq(Z A)^{\mathbf{\Omega}}$ onto a subalgebra $A \bar{\eta} \subseteq((Z A) / K)^{\Omega}$; we also denote $A \bar{\eta}$ by $A / K$ and call $\bar{\eta}(A \rightarrow A / K)$ the canonical homomorphism.

2.1. Let $\phi(A \rightarrow B)$ be a homomorphism of $A$ into $B(A, B \in L)$ with $r$-kernel $K_{\phi}$ and let $\eta\left(A \rightarrow A / K_{\phi}\right)$ be the canonical homomorphism. Then there exists a unique isomorphism $\phi\left(A / K_{\phi} \rightarrow B\right)$ such that $\eta \bar{\phi}=\phi$. (Compare (6), p. 130, Theorem 2.2).

Proof. If $\Gamma_{\phi}$ denotes the congruence on $A$ corresponding to $\phi$, then $\Gamma_{\phi}=\Gamma_{\eta}$, since if $(a, b) \in A \times A$ then

$$
\begin{aligned}
(a, b) \in \Gamma_{\phi} \Leftrightarrow a \phi=b \phi \Leftrightarrow(a-b) \phi^{*} & =0 \Leftrightarrow a-b \in K_{\phi} \\
\Leftrightarrow & a+K_{\phi}=b+K_{\phi} \Leftrightarrow a \eta=b \eta \Leftrightarrow(a, b) \in \Gamma_{\eta} .
\end{aligned}
$$

Like Theorem 2.1 of (6), p. 130, we can prove:

2.2. If $\left\{a_{i}\right\}_{i \in I}$ is a representative system of the congruence classes of $A \in L$ with respect to the congruence $\Gamma_{\phi}$ on $A$ corresponding to the homomorphism $\phi(A \rightarrow B)$, then

$$
M_{\phi}=\left\{a-a_{i} \mid a \in A,\left(a, a_{i}\right) \in \Gamma_{\phi}, a \neq a_{i}, i \in I\right\}
$$

is a Z-basis for the r-kernel $K_{\phi}$ of $\phi$.

Similarly Theorems 2.3 and 2.4 of (6), p. 131, can be extended to our case.

An ideal of $Z A$ which coincides with the $r$-kernel $K_{\phi}$ of a homomorphism $\phi(A \rightarrow B)$ we shall also call an $r$-kernel of $A$. From $(a, b) \in \Gamma_{\phi} \Leftrightarrow a-b \in K_{\phi}$ for all $a, b \in A$, together with 2.2 , we get

2.3. The correspondence $\Gamma_{\phi} \rightarrow K_{\phi}$ determines an isomorphism of the lattice of congruences on $A \in L$ onto the lattice of all r-kernels of $A$.

This proposition incidentally has an interesting application in the theory of semigroups. Since the lattice of congruences on $A$ can be imbedded in the 
lattice of ideals of $Z A$, we can prove directly Redei's theorem that any commutative semigroup $A$ which can be finitely generated is Noetherian: that is, $A$ satisfies the maximal condition for congruences. For if $A$ is generated by $n$ elements and $Z(x)$, where $[x]=\left[x_{1}, \ldots, x_{n}\right]$, is the polynomial ring in $n$ indeterminates over $Z$, then $Z A$ is a homomorphic image of the subring $R \subseteq Z[x]$ of all polynomials with zero constant term. Since every ideal of $R$ is also an ideal of the Noetherian ring $Z[x]$, then $R$ and therefore $Z A$ and $A$ are Noetherian. (Compare (1), p. 88).

\section{The closure operation $\mathrm{Cl}_{P}$}

We now make our prerequisites concerning the class $L$ more precise by adding that $L$ is to be closed under the operation of taking homomorphic images. Let $\Lambda$ denote the class of all surjective homomorphisms of algebras of $L$. For a natural number $n$, let $A^{n}$ denote the $n$th cross-product $A \times \ldots \times A$ and let $(Z A)^{n}$ denote $Z A \times \ldots \times Z A$.

With each algebra $A \in L$ we associate a function $P\left(A^{n} \rightarrow Z A\right)$ with the following property:

3.1. $\quad P\left(a_{1}, \ldots, a_{n}\right) \phi^{*}=P\left(a_{1} \phi, \ldots, a_{n} \phi\right)$

for all $a_{1}, \ldots, a_{n} \in A$ and all $\phi(A \rightarrow B) \in \Lambda$.

This condition is satisfied for example when for $P$ we choose a fixed polynomial $P\left(x_{1}, \ldots, x_{n}\right)$ in $n$ indeterminates $x_{1}, \ldots, x_{n}$, formed with the operations of $\Omega \cup\{+\}$. More generally, suppose that we define a function $P\left((Z A)^{n} \rightarrow Z A\right)$ as follows: let $\rho\left(x_{0}, x_{1}, \ldots, x_{n}\right)$ be a given positive formula in the first order predicate calculus with identity over the predicate domain $\Omega \cup\{+\}$. For all $Z A$ (such that $A \in L$ ) let

$$
\text { 3.2. } \wedge x_{1} \ldots \wedge x_{n} \vee x_{0} \rho\left(x_{0}, x_{1}, \ldots, x_{n}\right)
$$

be the defining condition, and let

$$
\text { 3.3. } \wedge x_{0} \wedge y_{0} \wedge x_{1} \ldots \wedge x_{n}\left(\rho\left(x_{0}, x_{1}, \ldots, x_{n}\right) \wedge \rho\left(y_{0}, x_{1}, \ldots, x_{n}\right) \Rightarrow x_{0}=y_{0}\right)
$$

be the condition of unambiguity. Then for all $s_{0}, s_{1}, \ldots, s_{n} \in Z A$, write

$$
s_{0}=P\left(s_{1}, \ldots, s_{n}\right) \Leftrightarrow \rho\left(s_{0}, s_{1}, \ldots, s_{n}\right) .
$$

This definition obviously remains meaningful when $s_{1}, \ldots, s_{n}$ are restricted to $A \subseteq Z A$ and similarly $\wedge x_{1} \ldots \wedge x_{n}$ in $\mathbf{3 . 2}$ and $\mathbf{3 . 3}$ are restricted to $A$, but in this case $P$ naturally has the form $P\left(A^{n} \rightarrow Z A\right)$. Again 3.1 is satisfied. The relation 3.1 is even valid for all homomorphisms $\phi$ of $A$ into $B(A, B \in L)$ when the universal quantifier $\wedge$ does not occur in the positive formula $\rho$. But, since we shall only consider surjective homomorphisms, this restriction is superfluous. On the other hand, our results are valid with certain modifications even for homomorphisms which are " into" if $\mathbf{3 . 1}$ is satisfied for such homomorphisms. (For example, in the statement of 3.6, = would be replaced by $\subseteq$ ).

Let $N$ be a subset of $A^{n}$ and let $\Delta_{P}(N)$ be the ideal of the ringoid $Z A$ (where 
$A \in L)$ generated by all elements of the form $P(a)$, where $(a)=\left(a_{1}, \ldots, a_{n}\right) \in N$. Evidently if $N=\varnothing$, then $\Delta_{\mathrm{P}}(N)=\{0\}$. Further, let

$$
C l_{P}(N)=\left\{(a) \in A^{n}, P(a) \in \Delta_{P}(N)\right\} .
$$

In particular, $C l_{P}(\varnothing)=I_{P}(A)={ }_{\text {Def }}\left\{(a) \in A^{n}, P(a)=\{0\}\right\}$. A subset $N \subseteq A^{n}$ is said to be $P$-closed whenever $N=C l_{P}(N)$. The following result can easily be verified.

3.4. $C l_{P}$ is a closure operation in the lattice $\mathscr{P}\left(A^{n}\right)$ of all subsets of $A^{n}$.

Thus the aggregate $\mathscr{V}_{P}(A)$ of all $P$-closed subsets of $A^{n}$ is a closure system of $\mathscr{P}\left(A^{n}\right)$ and so is a complete lattice. Its smallest element is $I_{P}(A)$ and its largest element is $A^{n}$. From the definitions of $\Delta_{P}(N)$ and $C l_{P}(N)$ we can immediately deduce the following:

3.5. The correspondence $N \rightarrow \Delta_{\mathrm{P}}(N)$ determines an isomorphic mapping of the lattice $\mathscr{V}_{\mathrm{p}}(A)$ into the lattice $\mathscr{I}(Z A)$ of ideals of the ringoid $Z A$.

Just as in Lemma 3.2 of (6), p. 132, we can prove:

3.6. For all $N \subseteq A^{n}$ and all $\phi(A \rightarrow B) \in \Lambda$,

$$
\left(\Delta_{P}(N)\right) \phi^{*}=\Delta_{P}\left(N \phi^{n}\right)
$$

Here $\phi^{n}\left(A^{n} \rightarrow B^{n}\right)$ is defined by

$$
\left(a_{1}, \ldots, a_{n}\right) \phi^{n}=\left(a_{1} \phi, \ldots, a_{n} \phi\right) .
$$

For $N \subseteq B^{n}$ we also define $N \phi^{-n}$ to be $N\left(\phi^{n}\right)^{-1} \subseteq A^{n}$.

Let $\psi(E \rightarrow F)$ be a monotone mapping of a partial ordering $E$ into a partial ordering $F$; in agreement with (9), (10), we call $\psi$ fully infimum-true or, more briefly, $\delta$-true, whenever for all subsets $M \subseteq E$ such that inf $M$ exists in $E$,

$$
\{y \in F, y \leqq x \psi \text { for all } x \in M\} \Rightarrow y \leqq(\inf M) \psi \text {. }
$$

Using this terminology the following proposition, which generalises Theorem 3.3 of (6), p. 132, can be deduced from 3.6.

3.7. For $\phi=\phi(A \rightarrow B) \in \Lambda$, the mapping of $\mathscr{P}\left(B^{n}\right)$ into $\mathscr{P}\left(A^{n}\right)$ given by $N \rightarrow N \phi^{-n}, N \subseteq B^{n}$, induces a $\delta$-true mapping of $\mathscr{V}_{P}(B)$ into $\mathscr{V}_{P}(A)$.

\section{4. $P$-factor algebras and $P$-kernels}

Given a $P$-closed subset $N \subseteq A^{n}$, we call $(A / N)_{P}={ }_{\text {Def }} A / \Delta_{P}(N)$ the $P$-factor algebra of $A$ with respect to $N$.

Given $\phi(A \rightarrow B) \in \Lambda$ we call $I_{P}(B) \phi^{-n}$ the $P$-kernel of $\phi$.

It follows from 3.7 that the $P$-kernel of $\phi$ is a $P$-closed subset of $A^{n}$. We observe that for all $(a) \in A^{n}$ and all $\phi(A \rightarrow B) \in \Lambda$ we have

$$
(a) \in I_{P}(B) \phi^{-n} \Leftrightarrow(a) \phi^{n} \in I_{P}(B) \Leftrightarrow P\left((a) \phi^{n}\right)=P(a) \phi^{*}=0 \Leftrightarrow P(a) \in K_{\phi} \cdot
$$

The homomorphism $\phi(A \rightarrow B) \in \Lambda$ is called $P$-true whenever the $P$-kernel of $\phi$ is equal to $I_{P}(A)$.

Given an ideal $K$ of $Z A$ let $\Delta K$ be the $Z$-submodule of $(Z A)^{+}$generated by 
all elements of $K$ of the form $a-b$, where $a, b \in A$. Clearly $\Delta K$ is also, like $K$, an ideal of $Z A$. It can easily be seen that $\Delta$ defines a closure operation in the lattice $\mathscr{I}(Z A)^{\cdot}$ dual to $\mathscr{I}(Z A)$. For the canonical mapping $\eta(A \rightarrow A / K)$ we obviously have $K_{\eta}=\Delta K$ and $A / K \cong A / K_{\eta}$. Conversely $K_{\phi}=\Delta K_{\phi}$ holds for all $\phi$. Thus given an ideal $K$ of $Z A$ the relation $K=\Delta K$ holds precisely when $K=K_{\phi}$ for some suitable $\phi=\phi(A \rightarrow B) \in \Lambda$.

4.1. For any $P$-closed subset $N \subseteq A^{n}$ the following statements are equivalent:

a) $\Delta_{P}(N)=\Delta \Delta_{P}(N)$

b) the P-kernel of the canonical homomorphism $\eta\left(A \rightarrow(A / N)_{P}\right)$ is equal to $N$.

Proof. $a) \Rightarrow b$ ). On the one hand

$$
(a) \in I_{P}\left(A / \Delta_{P}(N)\right) \eta^{-n} \Leftrightarrow P(a) \in K \eta=\Delta \Delta_{P}(N)
$$

and on the other hand

$$
(a) \in N \Leftrightarrow P(a) \in \Delta_{P}(N) .
$$

Thus, since $\left.\Delta_{P}(N)=\Delta \Delta_{P}(N), b\right)$ holds.

$b) \Rightarrow a)$. By hypothesis,

$$
P(a) \in \Delta \Delta_{P}(N) \Leftrightarrow P(a) \in \Delta_{P}(N)
$$

holds for all $(a) \in A^{n}$. Since this is true in particular for $(a) \in N$, and since $\Delta_{P}(N)$ is generated by all $P(a)$ where $(a) \in N$, it follows that

$$
\Delta_{P}(N)=\Delta \Delta_{P}(N) \text {. }
$$

The validity of the complicated condition 4.1 a) can be verified in many cases from the following sufficient and easily recognisable criterion.

4.2. Condition 4.1 a) is satisfied when for all $(a) \in A^{n}$ and all ideals $K$ of $Z A$ we have

$$
\text { a) } P(a) \in K \Rightarrow P(a) \in \Delta K
$$

This condition is equivalent to

$$
\text { b) } P(a) \in \Delta(P(a)) \text { for all }(a) \in A^{n} \text {, }
$$

where $(P(a))$ denotes the ideal of $Z A$ generated by $P(a)$ (principal ideal).

From 3.7 and 4.1 we get:

4.3. Assuming that 4.1 a) holds for all $N \in \mathscr{V}_{P}(A)$, the P-kernels are identical with the P-closed subsets of $A^{n}$.

Theorem 3.4 of (6), p. 132, which corresponds to our Proposition 4.1, is obviously incorrect since it omits the condition 4.1 a). For the same reason, all the theorems which follow from Theorem 3.4 in (6) hold only if condition $4.1 a$ ) is inserted. For example, from 4.1 we get:

4.4. Let $\phi(A \rightarrow B) \in \Lambda$ be a homomorphism with P-kernel $N$. Further let 4.1 a) be satisfied. 
Then there exists a unique P-true homomorphism (P-isomorphism)

$$
\bar{\phi}\left((A / N)_{P} \rightarrow B\right)
$$

such that $\eta \bar{\phi}=\phi$, where $\eta\left(A \rightarrow(A / N)_{P}\right)$ is the canonical homomorphism.

From 4.3 and 4.1 it follows that the requirements $a$ ) and $b$ ) made in the introduction are satisfied. The following proposition shows that requirement $c$ ) is also satisfied.

4.5. If every $N \in \mathscr{V}_{P}(A)$ satisfies condition 4.1 a), then the correspondence $\Gamma_{\phi} \rightarrow I_{P}(B) \phi^{-n}$, where $\phi(A \rightarrow B) \in \Lambda$, determines a $\delta$-true mapping of the lattice of congruences on $A$ into the lattice $\mathscr{V}_{P}(A)$.

Proof. From the relation

$$
I_{P}(B) \phi^{-n}=\left\{(a) \in A^{n}, P(a) \in K_{\phi}\right\}
$$

it follows that the mapping $\Gamma_{\phi} \rightarrow I_{P}(B) \phi^{-n}$ can be decomposed into

$$
\Gamma_{\phi} \rightarrow K_{\phi} \rightarrow I_{P}(B) \phi^{-n} \text {, }
$$

where $\Gamma_{\phi} \rightarrow K_{\phi}$ is an isomorphism, because of 2.3. Thus it is sufficient to show that the mapping $K_{\phi} \rightarrow I_{P}(B) \phi^{-n}$, where $\phi \in \Lambda$, is $\delta$-true. The monotonicity follows directly from the inclusion

$$
\left\{(a) \in A^{n}, P(a) \in K_{\phi_{1}}\right\} \subseteq\left\{(a) \in A^{n}, P(a) \in K_{\phi_{2}}\right\}
$$

where $K_{\phi_{1}} \subseteq K_{\phi_{2}}$ and $\phi_{1}\left(A \rightarrow B_{1}\right), \phi_{2}\left(A \rightarrow B_{2}\right) \in \Lambda$. Now let $\phi_{i}\left(A \rightarrow B_{i}\right) \in \Lambda$, where $i \in I$. Then we have

$$
\bigcap_{i \in I} K_{\phi_{i}} \rightarrow\left\{(a) \in A^{n}, P(a) \in \bigcap_{i \in I} K_{\phi_{i}}\right\}=\bigcap_{i \in I}\left\{(a) \in A^{n}, P(a) \in K_{\phi_{i}}\right\}
$$

\section{Examples}

In the following examples we assume that the class $L$ is always closed under homomorphic images.

5.1. Let $L$ be a class of algebras of type $\Omega$ and let $P(x, y)=x-y$. Clearly $P$ satisfies condition $4.2 a$ ). We assert that the $P$-closed subsets of $A \times A$ are precisely the congruences on $A$. This assertion was confirmed in (6), p. 134, by explicit calculation (in the case in which $L$ is the class of all groupoids). However the assertion is clear even without the calculation, since by 4.3 the $P$-closed sets are identical with the $P$-kernels. Now the smallest $P$-closed subset of $A \times A$ is the identity relation $O$. Thus if $\phi(A \rightarrow B) \in \Lambda$, then $O \phi^{-2}=\Gamma_{\phi}$ is $P$-closed.

If $N$ is a congruence on $A \in L$ and $\eta(A \rightarrow A / N)$ is the canonical mapping, then the kernel $K \eta$ of the homomorphism $\eta^{*}(Z A \rightarrow A(Z / N))$ consists precisely of the elements of the form $\sum_{i \in I} n_{i}\left(x_{i}-y_{i}\right)$, where $n_{i} \in Z$ and $\left(x_{i}, y_{i}\right) \in N$. Since $\Delta_{P}(N)$ is generated by the elements $x_{i}-y_{i}\left(\left(x_{i}, y_{i}\right) \in N\right)$, we have $\Delta_{P}(N)=K_{\eta}$ and $(A / N)_{P}=A / K_{\eta} \cong A / \Gamma_{\eta}=A / N$. 
5.2. Let $L$ be a class of ringoids of the type $\Omega_{0} \cup\left\{+_{0}\right\}=\Omega$, addition in a ringoid $A \in L$ being denoted by $+_{0}$. The corresponding algebra-ringoid $Z A$ (we avoid the word " ringoid-ringoid ") is then of the type

$$
\Omega \cup\{+\}=\Omega_{0} \cup\left\{+_{0}\right\} \cup\{+\} .
$$

For $A \in L$, write $P(x)=x-O_{A}$. Since condition $4.2 a$ ) is satisfied and $\left\{O_{A}\right\}$ is the smallest $P$-closed subset of $A$, the $P$-closed subsets are identical with the $P$-kernels, which coincide with the ideals of $A$.

Let $N$ be an ideal of $A$ and let $N^{\prime}$ be the congruence on $A$ corresponding to $N$, so that

$$
N^{\prime}=\left\{\left(a+_{0} b, b\right) \text {, where } a \in N, b \in A\right\} .
$$

As well as $P(x)=x-O_{A}$ we shall consider $P^{\prime}(x, y)=x-y$. Since $\Delta_{P}(N)$ and $\Delta_{P},\left(N^{\prime}\right)$ are the ideals of $Z A$ generated by all the elements of the forms $a-O_{A}$ and $\left(a+{ }_{0} b\right)-b$ respectively, where $a \in N, b \in A$, we have $\Delta_{P}(N) \subseteq \Delta_{P}\left(N^{\prime}\right)$. Since $a-O_{A} \in \Delta_{P}(N)$, we have

$$
\left(a-O_{A}\right)+{ }_{0} b=\left(a+{ }_{0} b\right)-\left(O_{A}+_{0} b\right)=\left(a+{ }_{0} b\right)-b \in \Delta_{P}(N) .
$$

Therefore we also have $\Delta_{P}(N) \supseteq \Delta_{P}\left(N^{\prime}\right)$ and so $\Delta_{P}(N)=\Delta_{P}\left(N^{\prime}\right)$. Thus $(A / N)_{P}=\left(A / N^{\prime}\right)_{P^{\prime}} \cong A / N={ }_{\text {Def }} A / N^{\prime}$.

5.3. Let $L$ be a class of algebras of finitary type $\Omega$. With each $A \in L$ associate an element $e_{A} \in A$ such that $e_{A} \phi=e_{B}$ for all $\phi(A \rightarrow B) \in \Lambda$. A nonempty subset $N \subseteq A$ is called normal ((8), p. 8), when for all polynomials $Q\left(x_{0}, x_{1}, \ldots, x_{m}\right)$ over $\Omega$ and all $a \in N, a_{1}, \ldots, a_{m} \in A$, it is true that

$$
Q\left(a, a_{1}, \ldots, a_{m}\right) \in N \Rightarrow Q\left(b, a_{1}, \ldots, a_{m}\right) \in N \text { for all } b \in N \text {. }
$$

From (8), p. 8, Theorem 5 , it follows that a subset $N \subseteq A$ is a congruence class of some congruence on $A$ precisely when $N$ is normal. A normal subset $N$ of $A$ will be called $e$-normal whenever $e_{A} \in N$. We write $P(x)=x-e_{A}$. Since $4.2 a$ ) is satisfied and $\left\{e_{A}\right\}$ is the smallest $P$-closed subset of $A$, the $P$-closed subsets of $A$ are identical with the $P$-kernels, which coincide with the $e$-normal subsets of $A$.

Given an $e$-normal subset $N \subseteq A$, let $N^{\prime} \subseteq A \times A$ be the Malcev congruence on $A$ corresponding to $N$ : that is, the transitive closure of the relation $(\sim)$ on $A$, with $x \sim y \Leftrightarrow x=y$, or $x, y \in N$, or there exists a polynomial $Q\left(x_{0}, x_{1}, \ldots, x_{m}\right)$ over $\Omega$ and elements $a_{1}, \ldots, a_{m} \in A$ such that $x, y \in Q\left(N, a_{1}, \ldots, a_{m}\right)$, where $Q\left(N, a_{1}, \ldots, a_{m}\right)={ }_{\operatorname{Def}}\left\{Q\left(a, a_{1}, \ldots, a_{m}\right)\right.$, where $\left.a \in N\right\}$. It is obvious that $N^{\prime}$ is the smallest congruence on $A$ with $N$ as a congruence class. If $P^{\prime}(x, y)=x-y$, we have $\Delta_{P}(N)=\Delta_{P}\left(N^{\prime}\right)$. For since $\Delta_{P}(N)$ is generated by all $a-e_{A}$ such that $a \in N$ and since $a \equiv e_{A} \bmod N^{\prime}$, then $\Delta_{P}(N) \subseteq \Delta_{P^{\prime}}\left(N^{\prime}\right)$. On the other hand the ideal $\Delta_{P}\left(N^{\prime}\right)$ is generated linearly over $Z$ from all $x-y$, where $x, y \in A$ and $x \sim y$. Now if $x \sim y$ and $x \neq y$, then either $x, y \in N$ and so $x-y=x-e_{A}-\left(y-e_{A}\right) \in \Delta_{P}(N)$, or there exist a polynomial $Q$ over $\Omega$ and $u, v \in N, a_{1}, \ldots, a_{m} \in A$ such that $x=Q\left(u, a_{1}, \ldots, a_{m}\right), y=Q\left(v, a_{1}, \ldots, a_{m}\right)$. 
From this it follows that

$$
\begin{aligned}
x-y & =Q\left(u, a_{1}, \ldots, a_{m}\right)-Q\left(e_{A}, a_{1}, \ldots, a_{m}\right)-Q\left(v, a_{1}, \ldots, a_{m}\right) \\
& +Q\left(e_{A}, a_{1}, \ldots, a_{m}\right) \\
& =Q\left(u-e_{A}, a_{1}, \ldots, a_{m}\right)-Q\left(v-e_{A}, a_{1}, \ldots, a_{m}\right) \in \Delta_{\mathrm{P}}(N),
\end{aligned}
$$

so that we have $\Delta_{P},\left(N^{\prime}\right) \subseteq \Delta_{P}(N)$. Thus equality holds. Since by $5.1 N^{\prime}$ is $P$-closed, then $N^{\prime}=\Gamma_{\eta^{\prime}}$, where $\eta^{\prime}\left(A \rightarrow(A / N)_{P^{\prime}}\right)$ is the canonical homomorphism. Since $\Delta_{P}(N)=\Delta_{P},\left(N^{\prime}\right)$, we have $(A / N)_{P}=\left(A / N^{\prime}\right)_{P}$, and so the canonical homomorphism $\eta\left(A \rightarrow(A / N)_{P}\right)$ coincides with $\eta^{\prime}$ and also $\Gamma_{\eta}=\Gamma_{\eta^{\prime}}=N^{\prime}$. From this it follows that

$$
\begin{aligned}
A / N & ={ }_{\text {Def }} A / N^{\prime}=A / \Gamma_{\eta} \cong A / K_{\eta}=A / \Delta \Delta_{P}(N) \\
& =A / \Delta_{P}(N)=(A / N)_{P} .
\end{aligned}
$$

5.4. Let $L$ be a class of groups and let $e_{A}$ be the unit element of $A \in L$. Then $e_{A} \phi=e_{B}$ for all $\phi(A \rightarrow B) \in \Lambda$. If we write $P(x)=x-e_{A}$, then by 5.3 the $P$-closed subsets are identical with the $P$-kernels, that is with the normal subgroups of $A$. Accordingly the $e$-normal subsets are precisely the normal subgroups of $A$ and $(A / N)_{P}$ is isomorphic to the factor group $A / N$ of $A$ with respect to the normal subgroup $N$ of $A$.

5.5. If $L$ is a class of semigroups with unit element $e_{A}$, where $A \in L$, then $e_{A} \phi=e_{B}$ for all $\phi(A \rightarrow B) \in \Lambda$. It follows from 5.3 that for $P(x)=x-e_{A}$ the concepts of $P$-closed subset, $P$-kernel and $e$-normal subset of $A$ coincide. It is obvious that a subset $N \subseteq A$ is $e$-normal precisely when $N$ is a normal subgroup of $A$ in the sense of semigroup theory (that is, for all $a, b \in A, x \in N$, we have $a x b \in N \Leftrightarrow a b \in N$ ). If $N$ is a normal subgroup of $A \in L$, then the Malcev congruence $N^{\prime}$ defined in $\mathbf{5 . 3}$ is identical with the Lyapin congruence on $A$ : that is, with the transitive closure of the relation $(\sim)$, where $x \sim y \Leftrightarrow x=y$ or $x, y \in a N b$ for suitable $a, b \in A$.

5.6. Let $L$ be a class of binary algebras (groupoids) with the binary operation written multiplicatively. We suppose that multiplication in $A \in L$ can always be carried out and that $A$ contains a zero element $O_{A}$ (so that $a O_{A}=O_{A} a=O_{A}$ for all $a \in A \in L)$. Then $O_{A} \phi=O_{B}$ for all $\phi(A \rightarrow B) \in \Lambda$. For $P(x)=x-O_{A}$ the $P$-kernels are precisely the ideals of $A$. It therefore follows from 5.3 that the concepts of $P$-closed subset, $P$-kernel, $O$-normal subset (with " $O$-normal " in place of " $e$-normal") and ideal of $A$ are identical. If $N$ is an ideal of $A$, then the corresponding Malcev congruence $N^{\prime}$ (as the smallest congruence with $N$ as a congruence class) is the Rees congruence on $A$ (that is $(x, y) \in N^{\prime} \Leftrightarrow x=y$ or $x, y \in N)$.

5.7. Let $L$ be a class of groupoids, which do not necessarily contain zero elements. Explicit calculation shows that for $Q(x)=x$ the $Q$-closed subsets are precisely the ideals of $A$ together with the empty set $\varnothing$. As $\varnothing$ is the smallest $Q$-closed set (it was apparently not considered in (6), p. 134), all $Q$-kernels are 
empty. This counter-example to Theorem 3.4 of (6), p. 132, shows at the same time that $4.1 a$ ) is certainly not true in general.

In the next section we shall introduce a new concept of kernel; it has the advantage that in the last example considered the $Q$-closed subsets and these alone appear as " kernels".

\section{Local parameters}

The parameter $P$ which occurs in our considerations so far satisfies the condition of uniformity 3.1. We shall now examine a more general case which can, for example, be realised by no longer using the same positive formula for all $A \in L$ for the definition of $P\left(A^{n} \rightarrow Z A\right)$, but by associating with any algebra $A$ its own positive formula $\rho_{A}$ subject to conditions $3.2,3.3$, along with a certain condition of connectivity with respect to $\rho_{A}, \rho_{B}, \ldots$.

We begin by assuming that a function $P_{A B}\left(B^{n} \rightarrow Z B\right)$ is associated with any pair $A, B \in L$ for which a surjective homomorphism $\psi(A \rightarrow B) \in \Lambda$ exists, in such a way that

6.1. $\quad P_{A A}\left(a_{1}, \ldots, a_{n}\right) \phi^{*}=P_{A B}\left(a_{1} \phi, \ldots, a_{n} \phi\right)$

for all $a_{1}, \ldots, a_{n} \in A$ and all $\phi(A \rightarrow B) \in \Lambda$.

Given a subset $N \subseteq B^{n}$, let $\Delta_{P_{A B}}(N)$ be the ideal of $Z B$ generated by all elements of the form $P_{A B}(b)$ with $(b) \in N$. Let $C l_{P_{A B}}(N), I_{P_{A B}}(B)$, the property of $N \subseteq B^{n}$ being $P_{A B}$-closed and the lattice $\mathscr{V}_{P_{A B}}(B)$ be defined as in section 3 . We also assume the following: holds.

6.2. If $P_{A B}$ is defined, the condition of connectivity $\mathscr{V}_{P_{B B}}(B) \subseteq \mathscr{V}_{P_{A B}}(B)$

The statements 3.4 and $\mathbf{3 . 5}$ remain true if $P$ is replaced by $P_{C A}$ (assuming that $P_{C A}$ is defined for $C, A \in L$ ). Analogous to 3.6 and 3.7 we have:

6.3. For all $N \subseteq A^{n}$ and all $\phi(A \rightarrow B) \in \Lambda$,

$$
\left(\Delta_{P_{A A}}(N)\right) \phi^{*}=\Delta_{P_{A B}}\left(N \phi^{n}\right) \text {. }
$$

6.4. If $\phi=\phi(A \rightarrow B) \in \Lambda$, then $N \rightarrow N \phi^{-n}$, where $N \subseteq B^{n}$, induces a $\delta$-true mapping of $\mathscr{V}_{P_{B B}}(B)$ into $\mathscr{V}_{P_{A A}}(A)$.

Given a $P_{A A}$-closed subset $N \subseteq A^{n}$, we call $(A / N)_{P}={ }_{\text {Def }} A / \Delta_{P_{A A}}(N)$ the $P$-factor algebra of $A$ with respect to $N$. Given $\phi(A \rightarrow B) \in \Lambda, I_{P_{B B}}(B) \phi^{-n}$ is called the $P$-kernel of $\phi$, and $\phi$ is called $P$-true when its $P$-kernel is equal to $I_{P_{A A}}(A)$. The analogue of 4.1 is:

6.5. For any $P_{A A^{-}}$closed subset $N \subseteq A^{n}$ the following statements are equivalent:

a) for all $(a) \in A^{n}$, we have

$P_{A A}(a) \eta^{*} \in \Delta_{P_{A, A \eta}}\left(I_{P_{A \eta, A \eta}}(A \eta)\right) \Leftrightarrow P_{A A}(a) \in \Delta_{P_{A A}}(N)$;

b) the P-kernel of the canonical homomorphism $\eta\left(A \rightarrow(A / N)_{P}\right)$ is equal to $N$. 
Proof. From 6.2 and 6.1 it follows that

$$
\begin{gathered}
(a) \in I_{P_{A \eta, A \eta}}(A \eta) \eta^{-n} \Leftrightarrow(a) \eta^{n} \in I_{P_{A \eta_{0}, A \eta}}(A \eta)=C l_{P_{A, A} \eta}\left(I_{P_{A \eta, A} \eta}(A \eta)\right) \\
\Leftrightarrow P_{A A}(a) \eta^{*}=P_{A}, A_{A}\left((a) \eta^{n}\right) \in \Delta_{P_{A, A},}\left(I_{P_{A \eta, A \eta}}(A \eta)\right) .
\end{gathered}
$$

From 6.4 and 6.5 we get

6.6. If 6.5 a) is satisfied for all $N \in \mathscr{V}_{P_{A A}}(A)$, then the P-kernels are identical with the P-closed subsets of $A^{n}$.

As a generalisation of 4.4 we obtain

6.7. Let $\phi(A \rightarrow B) \in \Lambda$ be a homomorphism whose P-kernel $N$ satisfies 6.5 a); moreover suppose that the condition

$$
\text { a) } \Delta_{P_{A B}}\left(I_{P_{B B}}(B)\right)=\{0\}
$$

is satisfied. Then there exists a unique P-true homomorphism $\phi\left((A / N)_{P} \rightarrow B\right)$ such that $\eta \bar{\phi}=\phi$, where $\eta\left(A \rightarrow(A / N)_{P}\right)$ is the canonical homomorphism.

6.8. If 6.5 $a$ ) is satisfied for all $N$ in the lattice $\mathscr{V}_{P_{A A}}(A)$, the correspondence $\Gamma_{\phi} \rightarrow I_{P_{B B}}(B) \phi^{-n}$, where $\phi(A \rightarrow B) \in \Lambda$, determines a monotone mapping of the lattice of congruences on $A$ into $\mathscr{V}_{P_{A A}}(A)$.

Proof. We consider the correspondence $K_{\phi} \rightarrow I_{P_{B B}}(B) \phi^{-n}$. If $\phi_{1}\left(A \rightarrow B_{1}\right)$, $\phi_{2}\left(A \rightarrow B_{2}\right) \in \Lambda$ and $K_{\phi_{1}} \subseteq K_{\phi_{2}}$, then $\Gamma_{\phi_{1}} \subseteq \Gamma_{\phi_{2}}$. Thus there exists a homomorphism $\psi\left(B_{1} \rightarrow B_{2}\right) \in \Lambda$ such that $\phi_{2}=\phi_{1} \psi$. By 6.4,

and

$$
I_{P_{B_{1} B_{1}}}\left(B_{1}\right) \subseteq I_{P_{B_{2} B_{2}}}\left(B_{2}\right) \psi^{-n}
$$

$$
I_{P_{B_{1} B_{1}}}\left(B_{1}\right) \phi_{1}^{-n} \subseteq I_{P_{B_{2} B_{2}}}\left(B_{2}\right) \psi^{-n} \phi_{1}^{-n}=I_{P_{B_{2} B_{2}}}\left(B_{2}\right) \phi_{2}^{-n} .
$$

In particular, if $K_{\phi_{1}}=K_{\phi_{2}}$, then $\Gamma_{\phi_{1}}=\Gamma_{\phi_{2}}$. In this case, $\psi$ is an isomorphism. By 6.4, $\psi^{-n}$ induces a monotone mapping of $\mathscr{V}_{P_{B_{2} B_{2}}}\left(B_{2}\right)$ into $\mathscr{V}_{P_{B_{1} B_{1}}}\left(B_{1}\right)$ and $\left(\psi^{-1}\right)^{-n}=\psi^{n}$ induces a monotone mapping of $\mathscr{V}_{P_{B_{1} B_{1}}}\left(B_{1}\right)$ into $\mathscr{V}_{P_{B_{2} B_{2}}}\left(B_{2}\right)$; therefore $\psi^{-n}$ induces an isomorphism of $\mathscr{V}_{P_{B_{2} B_{2}}}\left(B_{2}\right)$ onto $\mathscr{V}_{P_{B_{1} B_{1}}}\left(B_{1}\right)$, so that $I_{P_{B_{2} B_{2}}}\left(B_{2}\right) \psi^{-n}$ is equal to the smallest element $I_{P_{B_{1} B_{1}}}\left(B_{1}\right)$ of the lattice $\mathscr{V}_{P_{B_{1} B_{1}}}\left(B_{1}\right)$ From this it follows that

$$
I_{P_{B_{1} B_{1}}}\left(B_{1}\right) \phi_{1}^{-n}=I_{P_{B_{2} B_{2}}}\left(B_{2}\right) \phi_{2}^{-n}
$$

which shows that $K_{\phi} \rightarrow I_{P_{B B}}(B) \phi^{-n}$ unambiguously defines a mapping; also, by the inclusion proved previously, this mapping is monotone. Since $\Gamma_{\phi} \rightarrow K_{\phi}$ is certainly monotone, the same is true of the composition $\Gamma_{\phi} \rightarrow K_{\phi} \rightarrow I_{P_{B B}}(B) \phi^{-n}$.

We remark that in this general case (in contrast to 4.5), the $\delta$-trueness of the mapping given by $\Gamma_{\phi} \rightarrow I_{P_{B B}}(B) \phi^{-n}$ can only be proved given special prerequisites (compare, for example, (5), prop. 2.3).

The introduction of local parameters allows us to deal satisfactorily with the situation in Example 5.7 if $Q(x)=x$ is replaced by

$$
P_{A B}(x)=\left\{\begin{array}{l}
x-0_{B} \text { if } 0_{A} \in A \\
x \text { otherwise }
\end{array}\right.
$$


where $A, B \in L$ and $\phi(A \rightarrow B) \in \Lambda$ exists. (This definition is meaningful, since if $O_{A} \in A$, it follows that $O_{A} \phi=O_{B} \in B$ ). Then 6.1 is satisfied. We shall show that 6.2, 6.5 $b$ ) (and hence 6.5 $a$ )), and 6.7 $a$ ) are also satisfied.

From 5.6, $\mathscr{V}_{P_{A B}}(B)$ consists of all ideals of $B$ if $O_{A} \in B$ and from 5.7 it consists of all ideals of $B$ together with the empty set $\varnothing$ if $A$ does not have a zero element. Therefore whenever $P_{A B}$ is defined, $\mathscr{V}_{P_{B B}}(B) \subseteq \mathscr{V}_{P_{A B}}(B)$.

If $O_{A} \in A$ and $N \in \mathscr{V}_{P_{A A}}(A)$, then $P_{A A}(x)=x-O_{A}$,

$$
P_{A, A \eta}(x)=P_{A \eta, A \eta}(x)=x-O_{A \eta},
$$

where $\eta=\eta\left(A \rightarrow(A / N)_{P}\right)$, and by 4.1, 6.5 $\left.b\right)$ is equivalent to

$$
\Delta \Delta_{P_{A A}}(N)=\Delta_{P_{A A}}(N) \text {; }
$$

however, by 5.6, this equation is satisfied. Suppose therefore that $A$ has no zero element, so that $P_{A A}(x)=x$. Then $\mathscr{V}_{P_{A A}}(A)$ consists of all ideals and the empty subset $\varnothing$ of $A$. Given an ideal $N$ of $A$, we have

and therefore

$$
\Delta_{P_{A A}}(N)=\left\{\sum_{i \in I} n_{i} a_{i}, \text { where } n_{i} \in Z, a_{i} \in N\right\}
$$

$$
(a, b) \in \Gamma_{\eta} \Leftrightarrow a-b \in K_{\eta}=\Delta \Delta_{P_{A A}}(N) \Leftrightarrow a=b \text { or } a, b \in N .
$$

It follows that $\Gamma_{\eta}$ is the Rees congruence on $A$ and

$$
A / N={ }_{\text {Def }} A / \Gamma_{\eta} \cong A / K_{\eta}=A / \Delta \Delta_{P_{A A}}(N) \cong A / \Delta_{P_{A A}}(N)=A \eta .
$$

Since $N$ represents the zero element of $A / N$, we have $O_{A \eta} \in A \eta$ and

$$
P_{A \eta, A \eta}(x)=x-O_{A \eta} \text { and } I_{P_{A \eta, A \eta}}(A \eta)=\left\{O_{A \eta}\right\} \text {. }
$$

Hence for $a \in A$ we have

$$
a \in I_{P_{A \eta, A \eta}}(A \eta) \eta^{-1} \Leftrightarrow a \eta=O_{A \eta} \Leftrightarrow a \in N .
$$

Thus $6.5 b$ ) is satisfied. If $N=\varnothing$, then $\Delta_{P_{A A}}(N)=\{0\}$ and

$$
(a, b) \in \Gamma_{\eta} \Leftrightarrow a-b \in K_{\eta}=\{0\} \text {, }
$$

so that $a=b$ and $\eta$ is an isomorphism. Because $A \cong A \eta, A \eta$ does not have a zero element and therefore

and

$$
P_{A \eta, A \eta}(x)=x, \quad I_{P_{A \eta, A \eta}}(A \eta)=\varnothing
$$

$$
I_{P_{A \eta, A \eta}}(A \eta) \eta^{-1}=\varnothing=N .
$$

Thus $6.5 b$ ) is satisfied in all cases.

Let $A, B$ be a pair of algebras in $L$ for which there exists $\phi(A \rightarrow B) \in \Lambda$. If $O_{A} \in A$, then we also have $O_{B} \in B$ and $P_{A B}(x)=P_{B B}(x)=x-O_{B}$, so that

$$
\Delta_{P_{A B}}\left(I_{P_{B B}}(B)\right)=\Delta_{P_{B B}}\left(I_{P_{B B}}(B)\right)=\{0\} .
$$

If $A$ does not have a zero element, then $P_{A B}(x)=x$. If $B$ does not have a zero element, then it is also true that $P_{B B}(x)=x$ and that

$$
\Delta_{P_{A B}}\left(I_{P_{B B}}(B)\right)=\Delta_{P_{B B}}\left(I_{P_{B B}}(B)\right)=\{0\} \text {. }
$$


On the other hand, if $O_{B} \in B$, then $P_{B B}(x)=x-O_{B}, I_{P_{B B}}(B)=\left\{O_{B}\right\}$,

$$
\Delta_{P_{A B}}\left(\left\{O_{B}\right\}\right)=Z O_{B} \text { and } \Delta \Delta_{P_{A B}}\left(\left\{O_{B}\right\}\right)=\{0\} \text {. }
$$

\section{Category-theoretical aspect}

It is easily seen that

$$
\left\{A(\in L) \rightarrow Z A, \phi(A \rightarrow B)(\in \Lambda) \rightarrow \phi^{*}(Z A \rightarrow Z B)\right\}
$$

is a functor for the category $L, \Lambda$ into the category $L, \Lambda$ of all ringoids with the surjective homomorphisms as morphisms. Because of this we have a means of presenting the outlines of the preceding considerations in terms of category theory. In the present section we shall sketch this in the case of a (global) parameter $\boldsymbol{P}$. Certain postulates (F.1-F.8) are made which follow as closely as possible the earlier situation, although this in itself is not really essential.

Suppose that we are given two categories $L, \Lambda$ and $L, \Lambda$; here $L, L$ denote the classes of objects and $\Lambda, \Lambda$ the classes of morphisms. Since we are not at present interested in an intrinsic theory, but are merely drawing upon the categories to define certain functors, it does not matter whether they exist in concrete or abstract form. Let complete lattices $\mathscr{V}(A)$ and $\mathscr{V}(R)$ be associated with each object $A \in L$ and each $R \in L$ and suppose also that monotone mappings $\phi_{W}(\mathscr{W}(B) \rightarrow \mathscr{W}(A))$ and $\psi_{V}(\mathscr{V}(S) \rightarrow \mathscr{V}(R))$ are associated with each $\phi(A \rightarrow B) \in \Lambda$ and each $\psi(R \rightarrow S) \in \Lambda$. We define mappings $\phi_{W}^{*}(\mathscr{W}(A) \rightarrow \mathscr{W}(B))$ and $\psi_{V}^{*}(\mathscr{V}(R) \rightarrow \mathscr{V}(S))$ as follows:

$$
N \phi_{W}^{*}=\inf \left\{N^{\prime} \in \mathscr{W}(B), N \leqq N^{\prime} \phi_{W}\right\}, K \psi_{V}^{*}=\inf \left\{K^{\prime} \in \mathscr{V}(B), K \leqq K^{\prime} \psi_{V}\right\}
$$

where $N \in \mathscr{W}(A), K \in \mathscr{V}(R)$. Then for $\phi_{W}, \phi_{W}^{*}$ we have the following proposition, the proof of which is routine and can be omitted (compare (4)).

7.1. The following conditions are pairwise equivalent to one another:

(a) $N \leqq N \phi_{W}^{*} \phi_{W}$ for all $N \in \mathscr{W}(A)$;

(b) $\phi_{W}$ is $\delta$-true;

(c) $N \leqq N^{\prime} \phi_{W} \Leftrightarrow N \phi_{W}^{*} \leqq N^{\prime}$ for all $N \in \mathscr{W}(A), N^{\prime} \in \mathscr{W}(B)$;

(d) the pair $\phi_{W}\left(\mathscr{W}(B)^{*} \rightarrow \mathscr{W}(A)\right)$ and $\phi_{W}^{*}\left(\mathscr{W}(A) \rightarrow \mathscr{W}(B)^{\bullet}\right)$

is a Galois connexion, where $\mathscr{W}(B) \cdot$ denotes the lattice dual to $\mathscr{W}(B)$; tively.

(e) $\phi_{W}^{*} \phi_{W}$ and $\phi_{W} \phi_{W}^{*}$ are closure operations on $\mathscr{W}(A)$ and $\mathscr{W}(B)^{\bullet}$ respec-

There is, of course, a corresponding proposition involving $\psi_{V}, \psi_{V}^{*}$. It is well known that if $\phi_{W}$ (resp. $\psi_{V}$ ) is $\delta$-true, then $\phi_{W}^{*}\left(\right.$ resp. $\left.\psi_{V}^{*}\right)$ is fully supremumtrue or, more briefly $\sigma$-true ( $\sigma$-trueness and $\delta$-trueness are dual concepts). We postulate:

F.1. Let

$$
(w)=\left\{A(\in L) \rightarrow \mathscr{W}(A), \phi(\in \Lambda) \rightarrow \phi_{W}\right\}
$$


and

$$
(v)=\left\{R(\in L) \rightarrow \mathscr{V}(R), \psi(\in \Lambda) \rightarrow \psi_{V}\right\}
$$

be contravariant functors from $L, \Lambda$ and $L, \Lambda$ respectively into the category of all complete lattices, with the $\delta$-true mappings as morphisms.

Then we have the following:

7.2 .

$$
\left(\begin{array}{c}
* \\
w
\end{array}\right)=\left\{A(\in L) \rightarrow \mathscr{W}(A), \phi(\in \Lambda) \rightarrow \phi_{W}^{*}\right\}
$$

and

$$
\left(\begin{array}{l}
* \\
v
\end{array}\right)=\left\{R(\in L) \rightarrow \mathscr{V}(R), \psi(\in \mathbf{\Lambda}) \rightarrow \psi_{\mathrm{v}}^{*}\right\}
$$

are covariant functors of $L, \Lambda$ and $L, \Lambda$ respectively into the category of all complete lattices with the $\sigma$-true mappings as morphisms.

If $A \in L$ we define the star $\Lambda(A)$ to be the class of all $\phi(A \rightarrow B) \in \Lambda$ where $B \in L$. Similarly we define the $\operatorname{star} \Lambda(R)$ for $R \in L$. Regarding $L, \Lambda$ we make the following postulate:

F.2. For each $R \in \boldsymbol{L}$ let there be a "canonical " function $\kappa(\mathscr{V}(R) \rightarrow \boldsymbol{\Lambda}(R))$ and a "kernel" function $k(\Lambda(R) \rightarrow \mathscr{V}(R))$ such that the following diagram is commutative:

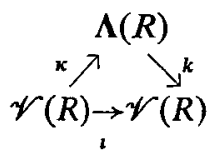

where $\imath$ denotes the identity.

For example if $\mathscr{V}(R)$ is the lattice of all factor objects (= quotient objects) $Q$ of an object $R$ of a bicategory $L, \Lambda$, then we can choose for $Q \kappa$ the projection $\pi: R \rightarrow Q$ in the sense of (7), $\left(11.1^{\prime}\right)$, p. 499 ; this is uniquely determined by $Q$.

Now let

$$
\left(^{1}\right)=\left\{A(\in L) \rightarrow A^{1}, \phi(\in \Lambda) \rightarrow \phi^{1}\right\}
$$

be a given covariant functor of $L, \Lambda$ into $L, \Lambda$. We require:

F.3. For all $A, B \in L, A^{1}=B^{1}$ implies that $A=B$. Moreover, for all $A \in L$ a correspondence $\left({ }^{0}\right)\left(\Lambda\left(A^{1}\right) \rightarrow \Lambda(A)\right)$ exists.

We also postulate:

F.4. There exists a natural transformation

$$
\left\{\pi_{A}\left(\mathscr{W}(A) \rightarrow \mathscr{V}\left(A^{1}\right)\right)\right\}_{A \in L}
$$

of the functor $\left(\begin{array}{l}* \\ W\end{array}\right)$ into the functor $\left({ }^{1}\right)\left(\begin{array}{l}* \\ V\end{array}\right)$.

This means that the mappings $\pi_{A}$ are $\sigma$-true and $\pi_{A}\left(\phi^{1}\right)_{V}^{*}=\phi_{W}^{*} \pi_{B}$ for all $\phi(A \rightarrow B) \in \Lambda$. We define mappings $\pi_{A}^{\prime}\left(\mathscr{V}\left(A^{1}\right) \rightarrow \mathscr{W}(A)\right)$ in terms of the mappings $\pi_{A}$ by putting

for all $K \in \mathscr{V}\left(A^{1}\right)$.

$$
K \pi_{A}^{\prime}=\sup \left\{N \in \mathscr{W}(A), K \geqq N \pi_{A}\right\}
$$

Analogous to 6.1 we have the following general result: 
7.3. For a monotone mapping $\pi_{A}\left(\mathscr{W}(A) \rightarrow \mathscr{V}\left(A^{1}\right)\right)$ the following conditions are equivalent:

a) $K \geqq K \pi_{A}^{\prime} \pi_{A}$ for all $K \in \mathscr{V}\left(A^{1}\right)$;

b) $\pi_{A}$ is $\sigma$-true;

c) $N \pi_{A} \leqq K \Leftrightarrow N \leqq K \pi_{A}^{\prime}$ for all $N \in \mathscr{W}(A), K \in \mathscr{V}\left(A^{1}\right)$;

d) the pair $\pi_{A}\left(\mathscr{W}(A) \rightarrow \mathscr{V}\left(A^{1}\right)^{\circ}\right), \pi_{A}^{\prime}\left(\mathscr{V}\left(A^{1}\right)^{\bullet} \rightarrow \mathscr{W}(A)\right)$

is a Galois connexion, where $\mathscr{V}\left(A^{1}\right)^{*}$ is the lattice dual to $\mathscr{V}\left(A^{1}\right)$;

e) $\pi_{A} \pi_{A}^{\prime}$ and $\pi_{A}^{\prime} \pi_{A}$ are closure operations in $\mathscr{W}(A)$ and $\mathscr{V}\left(A^{1}\right)^{\cdot}$ respectively,

From the fact that $\pi_{A}$ is $\sigma$-true, it follows that $\pi_{A}^{\prime}$ is $\delta$-true. Since $\pi_{A} \pi_{A}^{\prime}$ is a closure operation on $\mathscr{W}(A)$, the closed elements $N \in \mathscr{W}(A)$ (that is $N \pi_{A} \pi_{A}^{\prime}=N$ ) form a closure system $\mathscr{W}_{\pi \pi}(A)$ of $\mathscr{W}(A)$ and the correspondence

$$
N \rightarrow N \pi_{A}\left(N \in \mathscr{W}_{\pi \pi^{\prime}}(A)\right)
$$

is an anti-isomorphism of $\mathscr{W}_{\pi \pi^{\prime}}(A)$ onto the closure system $\mathscr{V}_{\pi^{\prime} \pi}\left(A^{1}\right)^{\cdot}$ of all elements of $\mathscr{r}\left(A^{1}\right)^{\cdot}$ closed with respect to $\pi_{A}^{\prime} \pi_{A}$. We have:

7.4. $\pi_{A}$ induces an isomorphism of $\mathscr{W}_{\pi \pi^{\prime}}(A)$ onto $\mathscr{V}_{\pi^{\prime} \pi}\left(A^{1}\right)$.

Since $N \pi_{A}\left(\phi_{V}^{1}\right)^{*}=N \phi_{W}^{*} \pi_{B}$ for all $N \in \mathscr{W}(A)$, we get

7.5. For $\phi(A \rightarrow B) \in \Lambda,\left(\phi^{1}\right)_{V}^{*}$ induces a $\sigma$-true mapping of $\mathscr{V}_{\pi^{\prime} \pi}\left(A^{1}\right)$ into $\mathscr{V}_{\pi^{\prime} \pi}\left(B^{1}\right)$.

We make the following requirement regarding the mappings $\left\{\pi_{A}^{\prime}\right\}_{A \in L}$ :

F.5. $\left\{\pi_{A}^{\prime}\left(\mathscr{V}\left(A^{1}\right) \rightarrow \mathscr{W}(A)\right)\right\}_{A \in L}$ is a natural transformation of the contravariant functors $\left({ }^{1}\right)(v)$ and $\left({ }_{W}\right)$.

This requirement means that the relation

$$
\pi_{B}^{\prime} \phi_{W}=\left(\phi^{1}\right)_{V} \pi_{A}^{\prime}
$$

is valid for all $\phi(A \rightarrow B) \in \Lambda$. From this we get

7.6. If $\phi(A \rightarrow B) \in \Lambda$, then $\phi_{W}$ induces a $\delta$-true mapping of $\mathscr{W}_{\pi \pi}$.(B) into $\mathscr{W}_{\pi \pi} \cdot(A)$.

We further postulate:

F.6. For the identity mapping $\imath(A \rightarrow A) \in \Lambda, \imath^{1} k$ is the smallest element of $\mathscr{V}\left(A^{1}\right)$.

F.7. For all $\phi(A \rightarrow B) \in \Lambda$, we have $\phi^{1} k=l^{1} k\left(\phi^{1}\right)_{V}$, where $\imath=\imath(B \rightarrow B)$ is the identity.

F.8. For all $N \in \mathscr{H}(A)$ the following implication holds:

E.M.S. -C

$$
N \pi_{A} \kappa^{01} k \pi_{A}^{\prime}=N \pi_{A} \pi_{A}^{\prime} \Rightarrow N \pi_{A} \kappa^{01} k=N \pi_{A} .
$$


We can now define the $\pi \pi^{\prime}$-factor algebra $(A / N)_{\pi \pi^{\prime}} \in L$ of $A \in L$, where $N \in \mathscr{W}_{\pi \pi^{\prime}}(A)$, by the mapping

$$
\eta\left(A \rightarrow(A / N)_{\pi \pi^{\prime}}\right)=N \pi_{A} \kappa^{0} \in \Lambda .
$$

Let the $\pi \pi^{\prime}$-kernel of $\phi(A \rightarrow B) \in \Lambda$ be defined in accordance with

$$
\operatorname{ker}_{\pi \pi^{\prime}} \phi=\phi^{1} k \pi_{A}^{\prime} \in \mathscr{W}_{\pi \pi^{\prime}}(A)
$$

If $I_{\pi \pi^{\prime}}(A)$ denotes the smallest element of the lattice $\mathscr{W}_{\pi \pi^{\prime}}(A)$, then we have:

7.7. $\operatorname{ker}_{\pi \pi^{\prime}} \phi=I_{\pi \pi^{\prime}}(B) \phi_{W}$ for all $\phi(A \rightarrow B) \in \Lambda$.

Proof. If $\imath(A \rightarrow A)$ is the identity for $A \in L$, then $\imath^{1}\left(A^{1} \rightarrow A^{1}\right)$ is the identity; by F.6, $\imath^{1} k$ is the smallest element of $\mathscr{V}\left(A^{1}\right)$ and is therefore the largest element of $\mathscr{V}\left(A^{1}\right)^{\cdot}$ and the closure system $\mathscr{V}_{\pi^{\prime} \pi}\left(A^{1}\right)^{\bullet}$. From 7.4 it follows that

$$
\iota^{1} k=I_{\pi \pi^{\prime}}(A) \pi_{A} \text { and } \imath^{1} k \pi_{A}^{\prime}=I_{\pi \pi^{\prime}}(A) .
$$

Now if $\phi(A \rightarrow B) \in \Lambda$, then F.5 and F.7 give

$$
I_{\pi \pi^{\prime}}(B) \phi_{W} \doteq \iota^{1} k \pi_{B}^{\prime} \phi_{W}=\iota^{1} k\left(\phi^{1}\right)_{V} \pi_{A}^{\prime}=\phi^{1} k \pi_{A}^{\prime}
$$

where $\imath=\imath(B \rightarrow B)$ is the identity.

7.8. For $N \in \mathscr{W}_{\pi \pi^{\prime}}(A)$, the following statements are equivalent:

a) $N \pi_{A} \kappa^{01} k=N \pi_{A}$;

b) $\operatorname{ker}_{\pi \pi^{\prime}} \eta\left(A \rightarrow(A / N)_{\pi \pi^{\prime}}\right)=N$.

Proof. $a) \Rightarrow b$ ). We have

$$
N=N \pi_{A} \pi_{A}^{\prime}=N \pi_{A} k^{01} k \pi_{A}^{\prime}=\eta^{1} k \pi_{A}^{\prime}=\operatorname{ker}_{\pi \pi}, \eta .
$$

b) $\Rightarrow a$ ). From $\operatorname{ker}_{\pi \pi^{\prime}} \eta=\eta^{1} k \pi_{A}^{\prime}=N$ we get

$$
N \pi_{A} \kappa^{01} k \pi_{A}^{\prime}=N=N \pi_{A} \pi_{A}^{\prime}
$$

and therefore, from F.8, $N \pi_{A} \kappa^{01} k=N \pi_{A}$.

We make the further observation that in many concrete cases the elements of the form $\phi^{1} k \in \mathscr{V}\left(A^{1}\right)$ form a complete lattice which is then mapped $\delta$-true onto the lattice $\mathscr{W}_{\pi \pi^{\prime}}(A)$ by $\phi^{1} k \rightarrow \operatorname{ker}_{\pi \pi^{\prime}} \phi=\phi^{1} k \pi_{A}^{\prime}$.

It can easily be seen that postulates F.1-F.8 are satisfied in the situation described in sections 2-5 if we choose for $L$ a class of algebras of type $\Omega$ closed under homomorphic images, with $\Lambda$ as the class of the corresponding surjective homomorphisms, for $\boldsymbol{L}, \boldsymbol{\Lambda}$ the category of all ringoids of type $\Omega \cup\{+\}$ with the corresponding surjective homomorphisms as morphisms, for $\left({ }^{1}\right)$ the functor $\left\{A(\in L) \rightarrow Z A, \phi(\in \Lambda) \rightarrow \phi^{*}\right\}$, for $\left({ }^{\circ}\right)$ the correspondence $\{\phi(Z A \rightarrow R)(\in \Lambda(Z A)) \rightarrow \phi \mid A$ (= the restriction of $\phi$ to $A \subseteq Z A)\}$, for ${ }_{W}$ ) the functor

$$
\left\{A(\in L) \rightarrow \mathscr{P}\left(A^{n}\right), \phi(\in \Lambda) \rightarrow \phi^{-n}\right\},
$$

for $(v)$ the functor $\left\{R(\in L) \rightarrow \mathscr{I}(R), \phi(\in \Lambda) \rightarrow \phi^{-1}\right\}$, and if for $K \in \mathscr{I}(R), K \kappa$ denotes the canonical homomorphism $\eta(R \rightarrow R / K)$ and for $\phi(R \rightarrow S) \in \Lambda, \phi k$ denotes the ideal $\{0\} \phi^{-1} \in \mathscr{I}(R)$. Here $\mathscr{I}(R)$ is the lattice of ideals of the ringoid 
HOMOMORPHISM THEORIES FOR UNIVERSAL ALGEBRAS 35

$R$. The mapping $\pi_{A}\left(\mathscr{P}\left(A^{n}\right) \rightarrow \mathscr{I}(Z A)\right)$ is defined by $N \pi_{A}=\Delta_{P}(N)$. Then $K \pi_{A}^{\prime}=\left\{(a) \in A^{n}, P(a) \in K\right\}$, where $K \in \mathscr{I}(Z A)$. For example, 4.1 and 7.8 then correspond to one another.

The observations of this section can also be applied more generally to cases in which $L, \Lambda$ and $L, \Lambda$ are categories of relational structures with surjective homomorphisms as morphisms.

\section{REFERENCES}

(1) L. Budach, Struktur Noetherscher kommutativer Halbgruppen, Monatsb. Deutsch. Akad. Wiss. Berlin, 6 (1964), 85-88.

(2) P. J. Hilton and W. Ledermann, Homology and ringoids. I, II, III, Proc. Cambridge Phil. Soc., 54 (1958), 152-167; 55 (1959), 149-164; 56 (1960), 1-12.

(3) Y. Hron, $\Omega$-ringoids, $\Omega$-rings and their representations, Colloq. Math., 14 (1966), 367-369.

(4) H.-J. HoenNKe, Über den Verband der Faktorobjekte (in the press).

(5) H.-J. HokhnKe, Das Brown-McCoysche Radikal für Algebren (in the press).

(6) G. Losey, A class of homomorphism theories for groupoids, Proc. Edinburgh Math. Soc., Ser. II, 14 (1964), 129-135.

(7) S. Maclane, Duality for groups, Bull. Amer. Math. Soc., 56 (1950), 485-516.

(8) A. I. Malcev, On the general theory of algebraic systems, Mat. Sb., 35 (77) (1954), 3-20 (Russian).

(9) J. SCHMidT, Abgeschlossenheits- und Homomorphiebegriffe in der Ordnungstheorie, Wiss. Z. Humboldt-Univ. Berlin, 3 (1953/54), 223-225.

(10) J. Schmid, Zur Kennzeichnung der Dedekind-MacNeilleschen Hülle einer geordneten Hülle, Arch. Math. (Basel), 7 (1956), 241-249.

Deutsche AKademie der Wissenschaften zU Berlin, FORSCHUNGSGEMEINSCHAFT, INSTITUT FÜR REINE MATHEMATIK. 